\title{
Halo Properties in Helium Nuclei from the Perspective of Geometrical Thermodynamics
}

M.C. Parker, School of Computer Sciences \& Electronic Engineering, University of Essex, Colchester C. Jeynes*, University of Surrey Ion Beam Centre, Guildford

W.N.Catford, Department of Physics, University of Surrey, Guildford

\section{Abstract}

The nuclear matter and charge radii of the helium isotopes $(A=4,6,8)$ are calculated by quantitative geometrical thermodynamics (QGT) taking as input the symmetry of the a-particle, the very weak binding (and hence halo nature) of the heavier helium isotopes, and a characteristic length scale given by the proton size. The results follow by considering each isotope in its ground state, with QGT representing each system as a maximum entropy configuration that conforms to the Holographic Principle. This allows key geometric parameters to be determined from the number of degrees of freedom available.

QGT treats ${ }^{6} \mathrm{He}$ as a ${ }^{4} \mathrm{He}$ core plus a concentric neutron shell comprising a holomorphic pair of neutrons, and the ${ }^{8} \mathrm{He}$ neutron halo is treated as a holomorphic pair of holomorphic pairs. Considering the information content of each system allows a correlation angle of $2 \square / 3$ between the holomorphic entities to be inferred, and then the charge radii of the three isotopes can be calculated from the displacement of the ${ }^{4} \mathrm{He}$ core from the centre of mass.

The calculations for the charge and matter radii of ${ }^{4,6,8} \mathrm{He}$ agree closely with observed values.

Similar QGT calculation of the sizes of the self-conjugate $A=4 n$ nuclei $\left\{{ }^{4} \mathrm{He},{ }^{8} \mathrm{Be},{ }^{12} \mathrm{C},{ }^{16} \mathrm{O},{ }^{20} \mathrm{Ne},{ }^{24} \mathrm{Mg},{ }^{28} \mathrm{Si},{ }^{32} \mathrm{~S},{ }^{36} \mathrm{Ar},{ }^{40} \mathrm{Ca}\right\}$ also agree well with experiment.

\section{Keywords}

Geometrical thermodynamics, holographic principle, halo nuclei, ${ }^{8} \mathrm{Be}$, dripline, ${ }^{10} \mathrm{He}$, Borromean

\section{Overview}

We will show that the matter and charge radii of the helium isotopes can be calculated ab initio purely from the geometry of the system, using the formalism of quantitative geometrical thermodynamics (QGT) developed by Parker \& Jeynes (2019: "PJ2019") ${ }^{1}$ and exploiting the holographic properties of maximum entropy ${ }^{2}$ (MaxEnt) structures (defined in Appendix 1). Note that the entropy description of a system by QGT is independent of (but isomorphic to) the familiar energy (kinematic) description of the system, with the system temperature transforming between the two descriptions (kinematic $\leftrightarrow$ entropic: see PJ2019 Table 1).

The alpha particle can be considered very simply in this approach as a unitary entity, and the weak binding of ${ }^{6,8} \mathrm{He}$ means that they can be considered as a ${ }^{4} \mathrm{He}$ core (a-particle) plus a halo of other holomorphic pairs of identical (neutron) entities. Our QGT treatment exploits this structural 
simplicity, leading to a simple formula for the entropy of the system in terms of the number of available degrees of freedom (DoFs). This number depends in essence upon the spatial symmetries of the system, a point discussed in more detail in Appendix 2. The Holographic Principle "places a fundamental limit on the number of degrees of freedom" 3 in any maximum entropy system.

These principles are applied to calculate $a b$ initio the matter radii of these various He nuclei. The charge radii for ${ }^{6} \mathrm{He}$ and ${ }^{8} \mathrm{He}$ are then calculated from the MaxEnt geometry of holomorphic (conjugated complex-vector) "trajectories". (In Appendix 1 the technical terms "holographic" and "holomorphic" are defined; note also that "trajectory" is used here in the particular geometric sense explained in connection with Figure 1.)

Starting from the geometric entropy behaviour for a single particle, we can learn about larger systems with certain geometric symmetries (composed of multiple particles) from purely QGT and MaxEnt considerations. We have applied that logic to two simple sets of systems: the helium isotopes ${ }^{4} \mathrm{He},{ }^{6} \mathrm{He}$ and ${ }^{8} \mathrm{He}$ discussed above, and the self-conjugate $\mathrm{A}=4 n$ nuclei $\left\{{ }^{4} \mathrm{He},{ }^{8} \mathrm{Be},{ }^{12} \mathrm{C},{ }^{16} \mathrm{O}, \ldots{ }^{40} \mathrm{Ca}\right\}$ which we will call the "He series". Both series are constructed in the QGT model from individual nucleons, and their nuclear sizes are uniquely determined in both series from a single characteristic scale length given by the size of the proton and assumed to be the same for both systems.

Curiously, the results agree with experimental measurements to an extraordinary precision. Table 1 and Table 2 respectively show measured and calculated nuclear radii for the He isotopes and the He series.

Briefly, the conceptual steps followed in the calculations are as follows:

i. the alpha particle is represented as a unitary (MaxEnt) entity in the QGT formalism wherein information and entropy ("info-entropy") are treated coherently;

ii. it is noted that the alpha particle, as a unitary (simplest possible) entity in its ground state, has in its rest frame just the 3 degrees of freedom (DoFs) required by 3-D space, and this then determines its entropy;

iii. the entropic formulation is then used via the Holographic Principle to construct geometric properties for both the He isotopes and the He series from the alpha particle, with further DoFs determined from the geometrical symmetries required by their MaxEnt properties.

The nuclear sizes follow in QGT from knowledge of the entropy, and the charge radii follow subsequently via the implied geometrical symmetry of the systems together with some simple arithmetic to evaluate the centre-of-mass motion. The system (in its ground state) corresponds to the minimum information (or equivalently the maximum entropy) and this is interpreted as meaning that geometrically the system exhibits the simplest allowed symmetry. In describing the alpha particles as "unitary entities" we do not refer to the technical mathematical term concerning the adjoint of Hermitian operators: instead, we mean those MaxEnt entities with no choice about their configuration which have the minimum number of available DoFs just as for "point 
particles" (that is, particles without either extension or internal DoFs). Holomorphic entities are unitary in this sense.

Quantitative geometrical thermodynamics is a systematic treatment of info-entropy: it is perhaps most surprising that information and entropy are conjugate orthogonal quantities, as shown already in mathematical detail by PJ2019 (their Eqs.2 and Appendix A; see also our Appendix 3). That is, information should not simply be identified as "neg-entropy" (negative entropy), as Léon Brillouin posited ${ }^{4}$. From this point of view it is seriously misleading to consider entropy merely as a measure of disorder: on the contrary, the operation of entropic forces can be powerful generators of order. As Ilya Prigogine pointed out in this context: "Recent developments in nonequilibrium physics and chemistry ... show unambiguously that the arrow of time is a source of order ... The entropy ... leads to an ordering process" ${ }^{5}$. Here we will see that a proper consideration of info-entropy has led us to a new understanding of the sizes of atomic nuclei. The alpha particle is seen to be a unitary entity, and the other self-conjugate $\mathrm{A}=4 n$ nuclei are seen as sums of two unitary entities. This is a remarkable result of an elementary QGT theory which at present handles only the static case. A fully dynamical theory based upon entropy production considerations can also be anticipated by explicitly invoking the time differential of entropy and employing the physics of entropy production already developed at length by Walter Grandy ${ }^{6,7}$. For example, an aspect of such a temporal development of QGT can already be seen in our expression for the entropic force (PJ2019 Eq.23).

\section{Borromean properties interpreted holomorphically}

The helium isotopes ${ }^{6} \mathrm{He}$ and ${ }^{8} \mathrm{He}$ are relatively long-lived, being particle-stable. Their half-lives are respectively $807 \mathrm{~ms}$ and $119 \mathrm{~ms}^{8,9}$ (decaying via $\square$ emission to ${ }^{6} \mathrm{Li}$ and ${ }^{8} \mathrm{Li}$ ). They are so-called 10 "halo nuclei" which have a neutron halo around a charged core. Thus, the root-meansquare (RMS) charge radius, measured as 2.068(11) fm and 1.929(26) fm for ${ }^{6} \mathrm{He}$ and ${ }^{8} \mathrm{He}$ respectively, ${ }^{11}$ is smaller than the RMS matter radius, measured as 2.33(4) fm and 2.49(4) fm respectively ${ }^{12}$. The measured ${ }^{4} \mathrm{He}$ charge radius and matter radius, respectively $1.676(8) \mathrm{fm}$ and $1.63(3) \mathrm{fm}$, are indistinguishable.

A bound system is said to be "Borromean" (after the Borromean rings of the $15^{\text {th }}$ century Casa Borromeo) if none of its sub-systems are bound. The nuclei ${ }^{6} \mathrm{He}$ and ${ }^{8} \mathrm{He}$ are regarded as Borromean since they are bound to particle emission but their subsystems $\left({ }^{5} \mathrm{He},{ }^{7} \mathrm{He}\right)$ are unbound and hence very short-lived $\left(<10^{-18} \mathrm{~s}\right)$ [ref.8]. Borromean binding has been demonstrated for the molecular hydrogen ion $\mathrm{H}_{2}^{+}$(with 2 protons and 1 electron an example ${ }^{13}$ of a 3-body system) while other nuclear examples include the ${ }^{11} \mathrm{Li}$ ground state ${ }^{14}$ along with the bound neutron-rich nuclei ${ }^{14} \mathrm{Be}$, ${ }^{19} \mathrm{~B}$ and others ${ }^{15}$.

The formalism of QGT and the related maximum entropy [ref.2] ("MaxEnt") techniques have been applied previously to explain the geometrical stability of DNA and the Milky Way galaxy (PJ2019). We will show that the He isotope stability has a similar origin in geometric entropy. 
Previously (in PJ2019) it was shown that the double logarithmic spiral (of which the double-helix is a special case) is a holomorphic structure. That is, its geometry conforms to the Cauchy-Riemann equations (of which Maxwell's equations are an important example in electro-magnetism) and is consequently represented correctly in an entropic Hamiltonian-Lagrangian formulation. PJ2019 also proved that the double-helix is a fundamental eigenvector of the entropic Hamiltonian, with its pitch and radius determining key properties. Separately, Parker \& Jeynes ${ }^{16}$ proved that a linear combination of two identical double-helices accounts for the stability of the spherical $\mathrm{C}_{60}$ (Buckminsterfullerene) molecule. They have also proved ${ }^{17}$ that the regular quantum operator representation follows directly from a proper (QGT) treatment of the entropic Liouvillian (see Appendix 4).

QGT is a systematic formulation of holomorphic info-entropy which has established the Principle of Least Exertion (PJ2019): this is the entropic isomorph of the kinematic Principle of Least Action. ${ }^{18}$ The natural consequence (shown by PJ2019) is that holomorphic objects are MaxEnt; that is, they are necessarily stable, having a most likely geometric configuration. In particular here, we demonstrate the existence of holomorphic descriptions of ${ }^{6} \mathrm{He}$ and ${ }^{8} \mathrm{He}$ nuclei. The lowest energy of a MaxEnt system such as ${ }^{6} \mathrm{He}$ has the minimum possible number of degrees of freedom, as any excited state would correspond to the utilisation of additional degrees of freedom. The theory then tells us the value of the maximum entropy that can be exhibited by the system in its lowest energy state.

Since entropy is essentially a scale-less quantity having units $(\mathrm{J} / \mathrm{K})$ independent of length (and time), and considering the universality of the laws of thermodynamics, we expect that the physics of entropy (and therefore also QGT) will apply throughout a diverse range of physical systems.

\section{Matter radii of He isotopes}

The nucleus ${ }^{4} \mathrm{He}$ (the alpha-particle) is a very simple and very stable system which has a particularly high binding energy between the four nucleons. This is inevitable quantum mechanically for a system containing two different families of identical particles (neutrons and protons) since $s_{1 / 2}$, the lowest energy orbital in a mean field, in ${ }^{4} \mathrm{He}$ is exactly filled with a pair of protons and a pair of neutrons. Combinations of multiple a-particles, the so-called selfconjugate nuclei with mass number $\mathrm{A}=4 n$ (with $n$ a positive integer), are also stable up to $\mathrm{A}=40$ (except the special case of ${ }^{8} \mathrm{Be}$ which is unbound). For convenience here, these nuclei are called the "helium series": ${ }^{4} \mathrm{He},{ }^{8} \mathrm{Be},{ }^{12} \mathrm{C},{ }^{16} \mathrm{O},{ }^{20} \mathrm{Ne},{ }^{24} \mathrm{Mg},{ }^{28} \mathrm{Si},{ }^{32} \mathrm{~S},{ }^{36} \mathrm{Ar},{ }^{40} \mathrm{Ca}$.

In the present QGT approach we represent ${ }^{4} \mathrm{He}$ as a unitary entity. In QGT the system entropy $S$ gains an amount $k_{\mathrm{B}}$ for each degree of freedom (DoF) that the system possesses: see Appendix 2 for the detailed demonstration that a unitary entity in 3-space has 3 DoFs so that the ${ }^{4} \mathrm{He}$ nucleus has an entropy:

$$
S_{4 \mathrm{He}}=3 k_{\mathrm{B}}
$$

where $k_{\mathrm{B}}$ is the Boltzmann constant. 
According to QGT we can also obtain the entropy $S$ of a system from its surface area $A$ and wavenumber $\square$ using an expression derived previously (see PJ2019: Eq.25, and Eq.D.15c in their Appendix D): the entropy of the single (double-spiral) eigenvector was given as:

$$
S_{\mathrm{DS}}=1 / 4 A \square^{2} k_{\mathrm{B}}
$$

where $\square$ is the system "wavenumber", and the system is encompassed (holographically) by the surface area given by $A$.

Wavenumber $\square$ is inversely proportional to wavelength $\square[\square$ and conventionally $\square \equiv 2 \square / \square \square \square$ But here, since we need only a parameter with the dimensions of length, we simply define the "holographic wavelength" $\square_{\mathrm{H}} \equiv 1 / \square \square$ so that we can write the general system entropy as:

$$
S=1 / 4 A k_{\mathrm{B}} / \square_{\mathrm{H}^{2}}^{2} .
$$

The values of $A$ and $\square_{\mathrm{r}}$ need to be determined for each system and can be understood to represent (respectively) the surface area and the entropic granularity or scale of the system. For example, in the context of the Bekenstein-Hawking equation for a black hole, $A$ is the area of the event horizon and $\square_{\mathrm{H}}$ is the Planck length $l_{\mathrm{P}}$. Eqs.2 are called "holographic" since the 3-D system property (here the entropy) is given by its surface area and not its volume. This is discussed further in Appendix 1, while we show elsewhere [ref.17] in a more fundamental treatment how to justify the Holographic Principle from the entropic Liouville theorem.

The surface area of the system corresponding to ${ }^{4} \mathrm{He}$ can be determined by equating the entropies given by equations (1) and (2b), once the holographic wavelength describing the length scale of the system has been chosen. The choice of this parameter is discussed below.

The nucleus ${ }^{6} \mathrm{He}$, in the present model, comprises the ${ }^{4} \mathrm{He}$ core plus a spherical "halo" of two identical neutrons. The halo is represented in QGT as two mutually holomorphic entities (neutrons), using the result proved by Parker \& Jeynes [ref.16] that a sphere may be represented holomorphically as the sum of two double-helices.

Note that the ${ }^{4} \mathrm{He}$ core is a unitary entity, that is, the simplest possible system (with no preferred axis), and the ${ }^{6} \mathrm{He}$ halo is an independent holomorphic system with its own (independentlyoriented) axis [ref.16]. That is to say, apart from sharing a common centre of spherical geometry, the two systems are not correlated geometrically.

Microscopic few-body models such as those described by Zhukov et al. (1993) ${ }^{19}$ or Bang et al. (1996) ${ }^{20}$ make the assumption that the weak binding of ${ }^{6} \mathrm{He}$ allows the two final neutrons to be treated as separate from the ${ }^{4} \mathrm{He}$ core and the same assumption is made here. Further, the (spherical) halo may be represented holomorphically since it comprises two identical objects (the neutrons) which together may form a (unitary) conjugate pair. Our QGT treatment treats the ${ }^{6} \mathrm{He}$ nucleus naturally as two conjoined (concentric) unitary entities. The ${ }^{6} \mathrm{He}$ nucleus (consisting of core and halo) therefore must have three further degrees of freedom over and above that of the ${ }^{4} \mathrm{He}$ core, to represent the relative orientation (in 3-space) of the halo, and thus:

$$
S_{6 \mathrm{He}}=6 k_{\mathrm{B}} \text {. }
$$


Note that in ${ }^{6} \mathrm{Li}$ the situation is different because the two additional particles need not act like identical particles. If they do act in such a way, then the state with the lowest possible energy is the $\mathrm{T}=1$ isotopic analogue of the ${ }^{6} \mathrm{He}$ ground state (which is the second excited state of ${ }^{6} \mathrm{Li}$ ). It is outside the scope of the present work to consider this further.

The nucleus ${ }^{8} \mathrm{He}$ comprises the ${ }^{4} \mathrm{He}$ core plus a spherical "halo" of now two holomorphic pairs of neutrons, corresponding respectively to an "inner" pair of neutrons for the ${ }^{6} \mathrm{He}$ shell and an extra "outer" pair of neutrons for the ${ }^{8} \mathrm{He}$ shell. Counting the pair of neutrons in the ${ }^{6} \mathrm{He}$ halo as a holomorphic entity (as just discussed), and now counting the extra pair of neutrons in the ${ }^{8} \mathrm{He}$ halo as another holomorphic entity (with the same holographic wavelength $\square_{\mathrm{H}}$ ), the four neutrons in the ${ }^{8} \mathrm{He}$ halo also together comprise a holomorphic pair of the two shells (again, with the same $\square_{\mathrm{H}}$ ). That is, a pair of identical holomorphic entities can itself be holomorphic, and where a holomorphic pair is formed the entropy is increased. Therefore, the MaxEnt condition will prefer holomorphic entities.

The requirement of MaxEnt implies minimum extra information. Howson \& $\mathrm{Urbach}^{21}$ make a clear statement to this effect: "Jaynes's [MaxEnt treatment] appeals...to the criterion...of minimum information: ...choos[ing]...the least information... or...making the fewest assumptions..." (see also Appendix 3). Alternatively, a MaxEnt configuration means that it is fully specified by the least information or fewest quantitative constraints. Therefore, MaxEnt dictates that these two pairs of neutrons must share the simplest common geometry for their holomorphic representations. The extra ("outer") 2-neutron halo of ${ }^{8} \mathrm{He}$ can be aligned in one of only two ways (i.e., "parallel" or "anti-parallel") to ${ }^{6} \mathrm{He}$. (In microscopic language, while the two pairs of additional neutrons in ${ }^{8} \mathrm{He}$ are separate from the core, they are able to interact with each other.)

Therefore the ${ }^{8} \mathrm{He}$ system must have only one additional degree of freedom, giving

$$
S_{8 \mathrm{He}}=7 k_{\mathrm{B}} .
$$

The surface area of the ${ }^{6} \mathrm{He}$ and ${ }^{8} \mathrm{He}$ systems can now be deduced just as for ${ }^{4} \mathrm{He}$. That is, equations (3) and (4) are combined with equation (2b) and we need only a value for the holographic wavelength, $\square \mathrm{H} \square \square$ We conjecture that the holographic wavelength is related to the proton, an entity forming the alpha particle. In any case, the results will scale with the wavelength and the suitability of any particular choice can be assessed by a comparison with the experimental data.

The observed proton and deuteron radii are given by CODATA2014 ${ }^{22}$ (Table XXXIII) as $r_{\mathrm{p}}=0.8751(61) \mathrm{fm}$ and $r_{\mathrm{d}}=2.1413(25) \mathrm{fm}$. A more recent (and accurate) value of $r_{\mathrm{d}}$ is $2.12562(78) \mathrm{fm} .{ }^{23}$ The deuteron in free space is a very weakly bound diffuse object (with a binding energy of about $1 \mathrm{MeV} /$ nucleon) whereas the four nucleons in an alpha particle are bound together with an energy of more than $7 \mathrm{MeV} /$ nucleon. The proton charge radius has been hotly debated since a smaller (and much more precise) value

$$
r_{\mathrm{pm}}=0.84087(39) \mathrm{fm}
$$


was obtained from muon scattering ${ }^{24}$. A new value from the Jefferson Laboratory "proton charge radius experiment" $(\mathrm{PRad})^{25}$ is

$$
r_{\text {PRad }}=0.831 \pm 0.007_{\text {stat }} \pm 0.012_{\text {syst }} \text { fm }
$$

obtained from e-p scattering; which confirms the muon scattering value and is also consistent with the updated value of the Rydberg constant adopted by CODATA2018.

We therefore choose a holographic wavelength $\square_{\mathrm{H}}$ for all the nuclear systems based on the alpha particle, including the nuclear halos of ${ }^{6,8} \mathrm{He}$ :

$$
\square_{\mathrm{H}}=2 \times r_{\mathrm{pm}}=1.682 \mathrm{fm}
$$

The surface area (and hence radius) of the sphere representing ${ }^{4} \mathrm{He}$ can now be obtained quantitatively by combining Eqs. (1) and (2b) and using the value in Eq.5b for $\square_{\mathrm{H}}$ :

$$
r_{4 \mathrm{He}}=\square_{\mathrm{H}} \times \sqrt{ }(3 / \square)=1.643 \mathrm{fm} .
$$

Similarly, using Eqs. (3) and (4) the matter radii for ${ }^{6} \mathrm{He}$ and ${ }^{8} \mathrm{He}$ can be computed:

$$
\begin{aligned}
& r_{6 \mathrm{He}}=\square_{\mathrm{H}} \times \sqrt{ }(6 / \square)=2.324 \mathrm{fm}, \\
& r_{8 \mathrm{He}}=\square_{\mathrm{H}} \times \sqrt{ }(7 / \square)=2.510 \mathrm{fm} .
\end{aligned}
$$

These values are compared with experiment in Table 1. It is immediately clear that the predicted radii from QGT show excellent agreement with the root mean square (RMS) matter radii determined experimentally. The comparison with the RMS radius is natural, because the radius of the entity is determined by its holographic area $A$ given in Eqs. 2: this system area represents a long-term average or equilibrium value since it comes from the thermodynamics. Similarly, the model of a holomorphic shell with a defined radius around a holomorphic core is a representation of the average behaviour of the system. The arithmetic of the small numbers of degrees of freedom means that the RMS matter radii are predicted to be in the simple numeric ratio ${ }^{4} \mathrm{He}:{ }^{6} \mathrm{He}:{ }^{8} \mathrm{He}$ of 1: $\sqrt{2}: \sqrt{7 / 3}$. The experimental values in Table 1 deviate from this by less than $1 / 2 \%$.

\section{The Symmetries of Halo Nuclei}

It is mandatory in QGT for a stable state to configure itself as maximum entropy (MaxEnt), given its energy. This necessarily corresponds to the state containing the minimum information. Therefore, the state of the system when considered geometrically should display the simplest possible symmetries, so that the number of rules determining that geometry is minimised.

We represent the geometry of the ${ }^{6} \mathrm{He}$ system as an alpha particle core plus two neutrons. These neutrons are identical (indistinguishable) fermions but their spin directions will be opposite, according to the Pauli principle which stipulates that the state of the system is fundamentally different upon exchange - that is, the wavefunction flips sign. Therefore exchanging the neutrons must result in a distinguishable configuration: this will turn out to be related to the symmetries involving reflection. 
The simplest geometric symmetry is rotation about an axis. QGT represents the ${ }^{6} \mathrm{He}$ and ${ }^{8} \mathrm{He}$ halos as spherical holomorphic entities with a well-defined axis (the $\underline{x}_{3}$ direction in Eqs.6 of Parker \& Jeynes, 2020 [ref.16]), which is required by QGT for such an entity. Note that reflection planes must include this axis, that is, the QGT symmetry groups do not include inversion: otherwise chiral QGT geometries (like DNA, see PJ2019 Fig.1) are excluded.

The one-fold geometrical symmetry has only a rotation by $2 p$ radians (the identity symmetry). Then satisfying reflection symmetry must be Pauli-forbidden (with respect to the plane defined by the 3-body system and the rotation axis), since this configuration is geometrically identical under exchange of the two neutrons. We therefore move on to consider higher order symmetries. For two-fold geometrical symmetry which is described by $\mathrm{D}_{2}$ the dihedral group of order 2, the alpha particle and two neutrons define a line. A rotation by $\mathrm{p}$ radians reproduces the spatial distribution along this line and is equivalent to exchanging the neutrons. However the system is ruled out, being symmetric under reflection in the plane through the line.

For three-fold geometrical symmetry, described by $\mathrm{D}_{3}$ (the dihedral group of order 3), a rotation by $2 \mathrm{p} / 3$ radians together with the corresponding reflections generates the symmetry group. The multiplication rules for this group represent the least amount of information that we can have, defining the geometrical symmetry of the system.

In order to invoke this $\mathrm{D}_{3}$ symmetry, the alpha particle and the two halo neutrons of ${ }^{6} \mathrm{He}$ are arranged with the two neutrons separated by an angle of $2 \mathrm{p} / 3$ and situated at the RMS matter radius deduced earlier for ${ }^{6} \mathrm{He}$. In fact, it's not obvious that this RMS radius is the one to use since the wavefunction of the neutron halo must extend considerably past it: we could consider a "halo radius" $r_{\mathrm{h}}$ to be given by $1 / 2\left(6 r_{\mathrm{h}}{ }^{2}+4 r_{\mathrm{c}}{ }^{2}\right)=r_{6 \mathrm{He}}{ }^{2}$, that is, $r_{\mathrm{h}}=3.26 \mathrm{fm}$, but this would impute a physical reality to the abstract quantity $r_{\mathrm{h}}$ : instead we use the observable RMS value for the nuclear matter radius $r_{6 \mathrm{He}}=2.32 \mathrm{fm}$ obtained above. An angle of $2 \mathrm{p} / 3$ radians then gives a "distance" (that is, an RMS distance) $r_{\mathrm{nn}}$ between the two halo neutrons of $4.02 \mathrm{fm}$, considering the halo neutrons to be located (on average) at radius $r_{6 \mathrm{He}}$.

Incidentally, the microscopic few-body calculation of Zhukov et al. (1993 [ref.19], their Table 7) gives $r_{6 \mathrm{He}}=2.59 \mathrm{fm}$ and $r_{\mathrm{nn}}=4.83 \mathrm{fm}$. Scaling to match the observed ${ }^{6} \mathrm{He}$ RMS matter $\operatorname{radius}\left(r_{6 \mathrm{He}}=2.32 \mathrm{fm}\right)$ would give a value of $r_{\mathrm{nn}}=4.33 \mathrm{fm}$ in the Zhukov model, which can be compared to $4.02 \mathrm{fm}$ as used here.

In order for the mean angle between the halo neutrons to be $2 p / 3$ radians, the distribution of probability at angles either side of that value needs to balance. The extreme value of $p$ radians corresponds to the two-fold symmetry that was rejected above as being impossible to exist on its own. This is the "cigar" configuration of Zhukov et al. Any peak in probability here could be balanced by a corresponding peak centred at $\mathrm{p} / 3$ radians. It is notable that the Zhukov model (using their values of $r_{\mathrm{nn}}=4.83 \mathrm{fm}$ and $r_{4 \mathrm{He}, \mathrm{n}}=4.43 \mathrm{fm}$ ) gives the angular separation of the neutrons at the nuclear surface as $66 \square(1.1 \times \mathrm{p} / 3$ radians $)$ and using their values of $r_{6 \mathrm{He}}=2.59 \mathrm{fm}$ and $r_{\mathrm{nn}}=4.83 \mathrm{fm}$ gives an overall average separation of $138 \square(1.15 \times 2 \mathrm{p} / 3$ radians $)$. The 
significance of this approximate agreement with three-fold symmetry is not entirely clear, but it is somehow reassuring that there appears to be no direct conflict between the microscopic models and the present QGT approach based on considerations of entropy, information and symmetry. The "dineutron" configuration identified by Zhukov et al. is connected to the peak in probability near $\mathrm{p} \beta$ radians. This configuration is shown in the model of Hagino et al. (2007) ${ }^{26}$ to exist mostly in the region of the nuclear surface, at least in the case of the similar Borromean nucleus ${ }^{11} \mathrm{Li}$, and experimental verification of this surface localisation has recently been forthcoming (Kubota et al., 2020) $)^{27}$.

The two coexisting "cigar" and "dineutron" configurations are closely related to the configurations with one-fold and two-fold symmetry that the above arguments imply cannot exist on their own, in a pure state. They can, apparently, coexist in a state with a geometry described on average by $\mathrm{D}_{3}$ symmetry. As a final comment about these two coexisting configurations, it is interesting to note that dynamical calculations for an orbiting two-body system give Lagrange points at which a third orbiting body will experience no net force. Relative to the first of two lighter bodies orbiting a heavier core, the second body experiences no net force at relative angles of $\mathrm{p} \beta$ radians (stable equilibrium) and $\mathrm{p}$ radians if it is on the same orbit. This is also reminiscent of the situation that is inferred here for ${ }^{6} \mathrm{He}$ and which also appears to come out of the microscopic models, although the forces involved in constraining the motion of the halo neutrons are completely different.

\section{Charge radii of the He isotopes}

Building on the QGT model's agreement with measurements of the RMS matter radii (Eqs.6), we extend the treatment by calculating the RMS charge radii.

The observed charge radius is larger for both ${ }^{6,8} \mathrm{He}$ than for ${ }^{4} \mathrm{He}$, and this is usually explained by spatial correlation of the halo neutrons giving a displacement of the centre of mass relative to the ${ }^{4} \mathrm{He}$ core. A reduction of the displacement for ${ }^{8} \mathrm{He}$ relative to ${ }^{6} \mathrm{He}$ indicates that the two pairs of neutrons are spatially anticorrelated. In our entropic representation this is readily modelled, ascribing a reality to the (abstract) geometrical description similar to that assumed in the conventional Glauber model ${ }^{28,29}$ for extracting nuclear sizes from cross-section data. Note that our QGT calculations, with an explicit integration over the time coordinate, are in some sense comparable to static probability distributions of quantum mechanics (yielding averages that would be obtained over time).

For ${ }^{6} \mathrm{He}$ the holomorphic pair of halo neutrons must have a simple and consistent relationship to each other, that is, the mean correlation angle $\square$ between them in Figure 1a must be fixed, so that they act as a unitary entity. The minimum number of degrees of freedom, means the condition for it representing a MaxEnt configuration (that is, the minimum - or least additional - information [21]) requires that the mean correlation angle $\square \square \square$ must be $\square=2 \square / 3$, as we saw in the last section. 


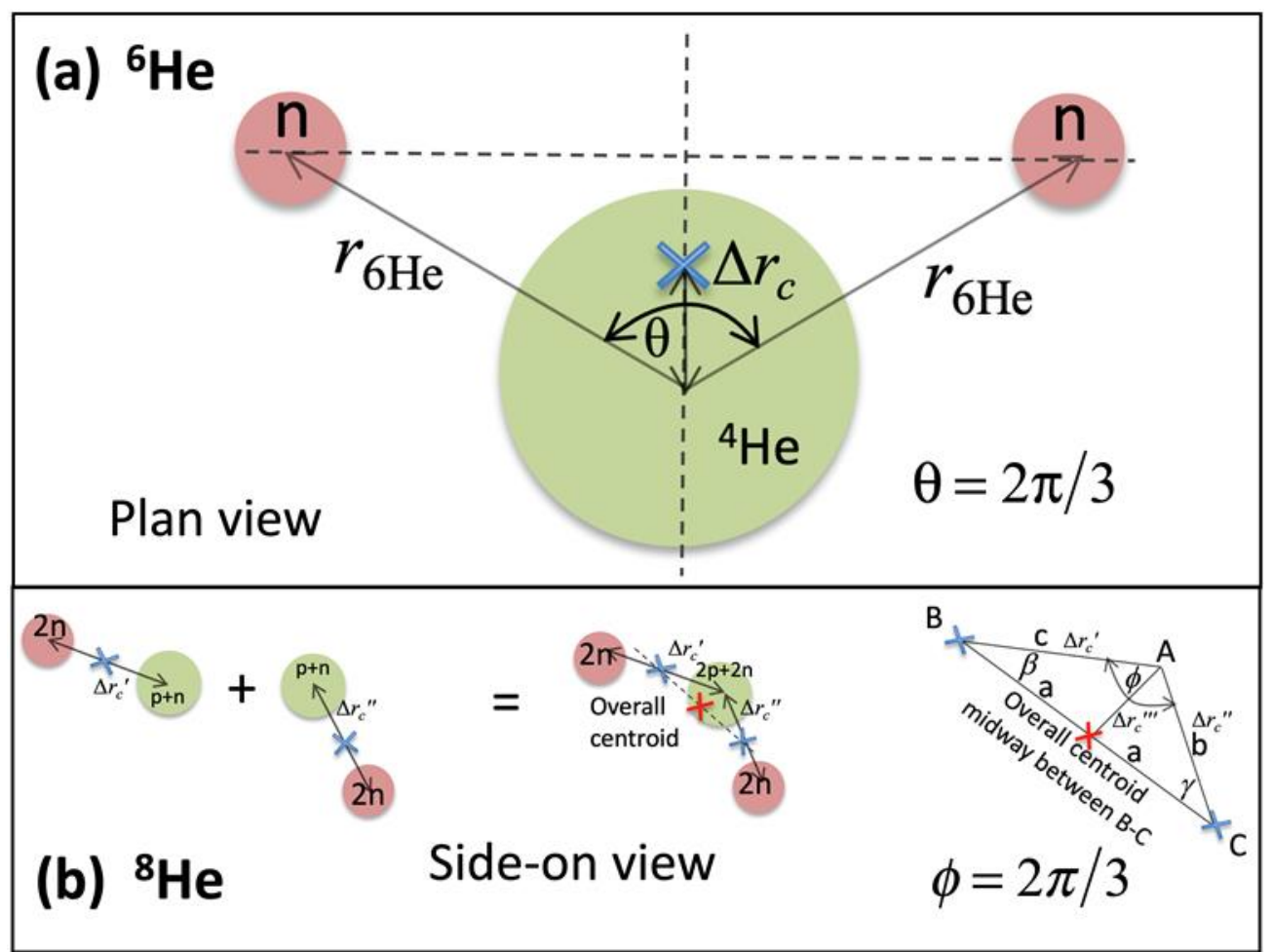

Figure 1: Calculation of the position of the centre of mass for ${ }^{6} \mathrm{He}$ and ${ }^{8} \mathrm{He}$.

a) $r_{6 \mathrm{He}}$ is assigned the value calculated for the RMS matter radius of ${ }^{6} \mathrm{He}$ and $\square r_{\mathrm{c}}$ is the displacement of the centre of mass from that of the ${ }^{4} \mathrm{He}$ core; $\boldsymbol{b}$ ) Analytic calculation of the geometric position of the centre of mass for ${ }^{8} \mathrm{He}$ is eased by assuming the overall system to simply comprise two subsystems of (i) half the alpha particle $\left({ }^{4} \mathrm{He}\right)$ core and two (inner halo) neutrons with a $\square r_{\mathrm{c}}^{\prime}$ displacement, and (ii) the other half-alpha core and two (outer halo) neutrons with a $\square r_{c}$ " displacement calculated using the matter radius of ${ }^{8} \mathrm{He}$. Combining (i) and

(ii) gives the overall displacement $\square r_{\mathrm{c}}{ }^{\prime \prime}$.

Here we treat the two neutrons of the ${ }^{6} \mathrm{He}$ halo as if it were literally true that they move in "trajectories" with a fixed angle to each other. Clearly, just as literal trajectories do not exist for elementary particles in a quantum mechanical description, such geometrical relations in the entropic description should be treated abstractly, comparing with time-averaged RMS values as for the matter radii. Also, this QGT treatment allows the wavefunction antisymmetry of the halo neutrons (in conformance to the Exclusion Principle) to naturally manifest itself as part of the overall MaxEnt condition.

The term 'trajectory' is not used here in the usual sense of 'position as a function of time', but as implying the underlying entropic formalism: that is, as a representation in 3-space of the evolution of the system in Minkowski 4-space where the 3-D geometrical representation is obtained by integrating over time in (4-D) Minkowski spacetime. The entropic 'trajectory' projects the effect of time onto the three dimensions of space by means of a contour integral in the complex 4-space (see PJ2019 Appendix A). Ultimately this represents the operation of the Second Law of Thermodynamics and therefore implicitly involves the arrow of time. The key differential variable $x_{3}$ in the QGT formalism (corresponding to the axis of the holomorphic geometry) is conjugate to the time variable $t \square x_{0} / c$. This indicates how a temporal trajectory is 
isomorphic to its spatial counterpart in the entropic geometry. Thus, 'trajectory' as used here is defined by the QGT formalism and represents a strictly geometrical (and not a temporal) view.

\begin{tabular}{|c|c|cc|cc|}
\hline Isotope & QGT & \multicolumn{2}{|c|}{ Matter Radius } & \multicolumn{2}{c|}{ Charge Radius } \\
& $\begin{array}{c}\text { DoF } \\
\text { Holographic }\end{array}$ & $\begin{array}{c}\text { Experimental } \\
\text { fm }\end{array}$ & fm & Holographic & Experimental \\
fm & fm \\
\cline { 2 - 6 } & $\square$ & 1.643 & $1.63(3)$ & 1.643 & $1.676(8)$ \\
${ }^{4} \mathbf{H e}$ & 3 & 2.324 & $2.33(4)$ & 2.031 & $2.068(11)$ \\
${ }^{\mathbf{6}} \mathrm{He}$ & 6 & 2.510 & $2.49(4)$ & 1.946 & $1.929(26)$ \\
${ }^{8} \mathbf{H e}$ & 7 & &
\end{tabular}

Table 1: Matter and charge radii for the helium isotopes.

The predictions of the holographic calculation (Eqs.6,7) are compared with the measured RMS values for the matter radii (see Table 2 of [ref.12]) and the charge radii (see Table III of [ref.11]). The number of

QGT degrees of freedom (DoF: D) in the holographic treatment is given for each isotope.

Then by symmetry, as the two neutrons (metaphorically) trace their trajectories, the locus of the centre of mass (indicated by a blue cross in Figure 1a) of the combined ${ }^{6} \mathrm{He}$ nucleus must lie on a sphere of radius $\square r_{c}$ compared to the radius of ${ }^{4} \mathrm{He}$ alone, and it is easy from the centroidal geometry to show that :-

$$
m_{4 \mathrm{He}} \times \square r_{\mathrm{c}}=2 m_{\mathrm{n}}\left(r_{6 \mathrm{He}} \cos \left(\square \square \square \square-\square r_{\mathrm{c}}\right)\right.
$$

where $m_{4 \mathrm{He}}$ and $m_{\mathrm{n}}$ are the masses of the ${ }^{4} \mathrm{He}$ (alpha particle) and a bound halo neutron respectively. In the centre of mass frame, the charge is therefore contained within a sphere of radius $r_{4 \mathrm{He}}+\square r_{\mathrm{c}}$. Then taking $\square=2 \square / 3$ as shown in Figure $2 \mathrm{a}$, and $4 m_{\mathrm{n}}=m_{4 \mathrm{He}}$ we have $\square r_{\mathrm{c}}=r_{6 \mathrm{He}} / 6=0.387 \mathrm{fm}$, giving a charge radius for ${ }^{6} \mathrm{He}:-$

$$
r_{6 \mathrm{He}}^{\text {charge }}=r_{4 \mathrm{He}}+\Delta r_{c}=2.031 \mathrm{fm}
$$

For ${ }^{8} \mathrm{He}$ we treat the two (holomorphic) pairs of halo neutrons as two concentric "inner" and "outer" shells, one with radius $r_{6 \mathrm{He}}$ and the other with radius $r_{8 \mathrm{He}}$ (see Figure 1b, with the displaced ${ }^{8} \mathrm{He}$ centre of mass indicated by a red cross) giving displacements for the two subsystems (with MaxEnt requiring $\square=2 \square / 3$ for both subsystems) of :-

$$
\begin{aligned}
& \square r_{\mathrm{c}}{ }^{\prime}=1 / 2 r_{6 \mathrm{He}} \cos \left(\square \square \square \square=r_{6 \mathrm{He}} / 4\right. \\
& \square r_{\mathrm{c}}{ }^{\prime}=1 / 2 r_{8 \mathrm{He}} \cos \left(\square \square \square=r_{8 \mathrm{He}} / 4 .\right.
\end{aligned}
$$

Then as shown in Figure 1b, with the additional requirement that the two pairs of halo neutrons also maintain a constant (MaxEnt) relative phase (or correlation angle) of $f=2 \square / 3$ between them, simple geometry gives $\square r_{\mathrm{c}}{ }^{\prime \prime}=0.303 \mathrm{fm}$, with a charge radius for ${ }^{8} \mathrm{He}$ then given by:

$$
r_{8 \mathrm{He}}^{\text {charge }}=r_{4 \mathrm{He}}+\Delta r_{c}^{\prime \prime}=1.946 \mathrm{fm}
$$

These values are compared with experiment in Table 1.

\section{The Case of ${ }^{10} \mathrm{He}$}

In the preceding treatment of ${ }^{6} \mathrm{He}$, a pair of holomorphic neutrons was added to a ${ }^{4} \mathrm{He}$ core (itself a unitary entity). In ${ }^{8} \mathrm{He}$ an additional pair of holomorphic neutrons was added, giving two identical holomorphic pairs able, in turn, to form a holomorphic pair. When we add a further pair to make ${ }^{10} \mathrm{He}$, the situation is different because there is nothing with which the additional holomorphic pair 
of neutrons can subsequently pair. Holomorphism is of pairs, not triples. The two extra neutrons must perforce remain separate and hence they correspond to a new spherical shell, not locked in orientation to the previous neutron shells. This must introduce a further three degrees of freedom (that is, equivalent to the entropic progression from ${ }^{4} \mathrm{He}$ to ${ }^{6} \mathrm{He}$ ):

$$
\begin{aligned}
& S_{10 \mathrm{He}}=10 k_{\mathrm{B}} \\
& r_{10 \mathrm{He}}=\square \mathrm{H} \times \sqrt{ }(10 / \square)=3.001 \mathrm{fm} .
\end{aligned}
$$

Experimentally, ${ }^{10} \mathrm{He}$ has been observed to be unbound to neutron emission with the ground state identified with a resonance in the continuum, as discussed, for example, by Parfenova $(2018)^{30}$. The simplest interpretation is that the additional size forced upon ${ }^{10} \mathrm{He}$ by the additional $3 k_{\mathrm{B}}$ of entropy is such that the final two neutrons are on average too far distant from the core to remain bound. This is speculation, but it is certainly true that ${ }^{10} \mathrm{He}$ would be required to be significantly larger, if it were to exist. The additional $0.5 \mathrm{fm}$ in RMS radius for ${ }^{10} \mathrm{He}$ in relation to ${ }^{8} \mathrm{He}$ is comparable with the additional $0.7 \mathrm{fm}$ introduced between the strongly bound ${ }^{4} \mathrm{He}$ and the justbound ${ }^{6} \mathrm{He}$, making an unbound ${ }^{10} \mathrm{He}$ a likely consequence.

To date, ${ }^{12} \mathrm{He}$ has not been observed, but we would expect it to be to ${ }^{10} \mathrm{He}$ as ${ }^{8} \mathrm{He}$ is to ${ }^{6} \mathrm{He}$, that is, to have one extra degree of freedom $\left(S_{12 \mathrm{He}}=11 k_{\mathrm{B}}\right)$ giving :-

$$
r_{12 \mathrm{He}}=\square_{\mathrm{H}} \times \sqrt{ }(11 / \square)=3.147 \mathrm{fm} .
$$

Note that ${ }^{8} \mathrm{He}$ (with a two-neutron separation energy, $\mathrm{S}_{2 \mathrm{n}}=2.14 \mathrm{MeV}$ ) is actually more strongly bound than ${ }^{6} \mathrm{He}\left(\mathrm{S}_{2 \mathrm{n}}=0.98 \mathrm{MeV}\right)$. Given that the RMS radius increases only weakly between these two isotopes, the increased number of neutron interactions can naturally cause this, and similarly in ${ }^{12} \mathrm{He}$.

\section{Matter radii for the "helium series"}

It is natural to seek further nuclear systems to test the QGT approach. The ${ }^{4} \mathrm{He}$ example and the

${ }^{6,8} \mathrm{He}$ extensions were the simplest place to begin, because of the simple way that they could be built entirely from pairs of identical components. In fact, the case of ${ }^{6} \mathrm{Be}$ is identical to ${ }^{6} \mathrm{He}$ in that respect, and the prediction for its size is thus identical, but ${ }^{6} \mathrm{Be}$ is unbound and exists as a resonant state only. Therefore, the QGT predictions are to be understood as implying that if the forces acting within the system are sufficient for it to form a localized bound system, then the size should be as predicted. Evidently, in ${ }^{6} \mathrm{Be}$ the additional Coulomb repulsion is sufficient to tip the balance between the weakly-bound halo of ${ }^{6} \mathrm{He}$ and a completely unbound system.

Interestingly, for ${ }^{6} \mathrm{Li}$ the analog of the ${ }^{6} \mathrm{He}$ ground state exists at an excitation energy of $3.56 \mathrm{MeV}$ $\left(\mathrm{J} \leftarrow=0^{+}, \mathrm{T}=1\right)$ and has the same wavefunction as ${ }^{6} \mathrm{He}$ but with a proton outside the ${ }^{4} \mathrm{He}$ core that acts as though it were a neutron (this excited state of ${ }^{6} \mathrm{Li}$ is also unbound). That is, its spin must be opposite to that of the neutron outside of the core, as if it were an identical particle. For the ground state of ${ }^{6} \mathrm{Li}$, the same holomorphic approach as above is not directly applicable because the additional proton and neutron are not identical and cannot form a comparable holomorphic pair. In fact, the observed RMS matter radius for the ground state of ${ }^{6} \mathrm{Li}, 2.551(31) \mathrm{fm}$, is 
consistent with $7 \mathrm{DoFs}$ (giving a calculated radius of $2.511 \mathrm{fm}$ : see Table 2 for $\mathrm{D}=7$ ). Thus the beta decay of ${ }^{6} \mathrm{He}$ as it transitions to ${ }^{6} \mathrm{Li}$ correlates with the addition of a DoF to the resulting nucleus, and the increase in system entropy expected from the $2^{\text {nd }}$ Law.

The next easiest case is actually ${ }^{8} \mathrm{Be}$, which could be a holomorphic pair of a-particles. To go from ${ }^{4} \mathrm{He}$ to ${ }^{8} \mathrm{Be}$ would then be analogous to the holomorphic addition of a second identical neutron pair to ${ }^{6} \mathrm{He}$ (i.e. it would introduce one additional degree of freedom, DoF) and thus ${ }^{8} \mathrm{Be}$ would have 4 DoFs. In this case (see Eqs.6) $r_{8 \mathrm{Be}}$ would be given by $\square_{\mathrm{H}} \times 2 / \sqrt{\square}=1.9 \mathrm{fm}$ (cf. $1.6 \mathrm{fm}$ for ${ }^{4} \mathrm{He}$ ) with a nuclear density of $0.279 \mathrm{amu} / \mathrm{fm}^{3}$, which is $30 \%$ higher than that of ${ }^{4} \mathrm{He}$ (density $0.215 \mathrm{amu} / \mathrm{fm}^{3}$ ). Since the alpha nucleus is already very tightly bound this increase in density looks impossible: of course, microscopically we know it is strictly Pauli-forbidden. A deeper QGT treatment than presented here would assert the same impossibility.

\begin{tabular}{|c|c|c|c|c|c|c|c|}
\hline \multirow{3}{*}{ Isotope } & \multirow{3}{*}{ A } & \multirow{3}{*}{$\begin{array}{c}\text { QGT } \\
\text { DoF } \\
\square \\
\end{array}$} & \multicolumn{3}{|c|}{ Measured } & \multicolumn{2}{|c|}{ Calculated (QGT) } \\
\hline & & & \multicolumn{2}{|c|}{ Radius } & \multirow{2}{*}{$\begin{array}{l}\text { RHW } \\
\square_{\mathrm{m}} / \square_{\mathbf{H}}\end{array}$} & \multirow{2}{*}{$\begin{array}{l}\text { Radius } \\
r_{\mathrm{A}}(\mathrm{fm})\end{array}$} & \multirow{2}{*}{$\begin{array}{l}\text { Density } \\
\text { amu/fm }{ }^{3}\end{array}$} \\
\hline & & & $r_{\mathrm{m}}(\mathrm{fm})$ & $\square r_{m}(a m)$ & & & \\
\hline${ }^{4} \mathrm{He}$ & 4 & 3 & 1.676 & 3 & 1.020 & 1.644 & 0.215 \\
\hline${ }^{8} \mathrm{Be}$ & 8 & 6 & "2.2" & & & 2.324 & 0.152 \\
\hline${ }^{12} \mathrm{C}$ & 12 & 7 & 2.470 & 2 & 0.984 & 2.511 & 0.181 \\
\hline${ }^{16} \mathrm{O}$ & 16 & 8 & 2.706 & 8 & 1.008 & 2.684 & 0.198 \\
\hline${ }^{20} \mathrm{Ne}$ & 20 & 9 & 3.005 & 4 & 1.056 & 2.847 & 0.207 \\
\hline${ }^{24} \mathrm{Mg}$ & 24 & 10 & 3.058 & 2 & 1.019 & 3.001 & 0.212 \\
\hline${ }^{28} \mathrm{Si}$ & 28 & 11 & 3.123 & 3 & 0.992 & 3.147 & 0.214 \\
\hline${ }^{32} S$ & 32 & 12 & 3.252 & 6 & 0.989 & 3.287 & 0.215 \\
\hline${ }^{36} \mathrm{Ar}$ & 36 & 13 & 3.391 & 6 & 0.991 & 3.422 & 0.215 \\
\hline${ }^{40} \mathrm{Ca}$ & 40 & 14 & 3.477 & 1 & 0.979 & 3.551 & 0.213 \\
\hline
\end{tabular}

Table 2: Helium series with nuclear radii measured, and calculated holographically by QGT Measured RMS charge radii $r_{\mathrm{m}}$ from Angeli $1999^{31}$, with the uncertainty $\square r_{\mathrm{m}}$ in attometres (this is a "last figure error" estimate) of the measurement; the "radius" of ${ }^{8} \mathrm{Be}$ is inferred from the calculations reviewed by Tohsaki $2017^{32}$ (and their Fig.4): see text; the relative holographic wavelength (RHW) is obtained from Eq.9b; calculated radii $r_{\mathrm{A}}$ from Eq.9a using the appropriate number of degrees of freedom (DoF) $\square$; the calculated nuclear density $\left(\mathrm{amu} / \mathrm{fm}^{3}\right)$ is derived from the calculated radius $r_{\mathrm{A}}$.

In order to build the effect of the Pauli Principle into the present model, we treat ${ }^{8} \mathrm{Be}$ like ${ }^{6} \mathrm{He}$, that is, we suppose it is two unitary entities that are dissimilar in the sense that they do not form a holomorphic pair. That is, ${ }^{8} \mathrm{Be}$ has (like ${ }^{6} \mathrm{He}$, and for the same reasons) $6 \mathrm{DoFs}$, and is therefore predicted to have the same RMS radius as ${ }^{6} \mathrm{He}$. Its nuclear density is listed in Table 2. Experimentally it is known that ${ }^{8} \mathrm{Be}$ is unbound, but its shape has nevertheless been measured, as noted by Bishop et al. (2019) ${ }^{33}$ : "The lightest (nontrivial) $\alpha$-conjugate system, ${ }^{8} \mathrm{Be}$, has been shown to have a structure comprising of a dumbbell configuration of $\alpha$ particles with a large $\alpha$ $\alpha$ separation distance of $4.4 \mathrm{fm}$ ". Since this value is rather roughly estimated from calculations, Table 2 simply uses half this distance for the radius. Since ${ }^{8} \mathrm{Be}$ is not spherical the proper measure involves the surface area of the (holographic) ellipsoid enclosing the nucleus, which would give a similar value for the RMS radius. 
When we continue up the "helium series" (the self-conjugate $\mathrm{A}=4 n$ nuclei), we note that each step adds one DoF for the same reason that ${ }^{8} \mathrm{He}$ has one more DoF than ${ }^{6} \mathrm{He}$ (see Appendix 2 for a discussion of "ontological DoFs"). That is, we treat all these nuclei as MaxEnt nuclei where the radius $r_{\mathrm{A}}$ is given as usual by the number of DoFs ( $\square$ in Table 2) for the nucleus, together with the holographic wavelength $\square_{\mathrm{H}}$ which is chosen to be the same as before (see Eq.5b):

$$
r_{\mathrm{A}}=\square_{\mathrm{H}} \times \sqrt{ }(\square \square / \square)
$$

Conversely, the holographic wavelength $\square_{\mathrm{m}}$ of the system can be determined ("measured") from the measured radius $r_{\mathrm{m}}$ :-

$$
\square \mathrm{m}=r_{\mathrm{m}} / \sqrt{ }(\square \square / \square)
$$

\section{Discussion}

We have derived, ab initio in a simple analytical treatment based on quantitative geometrical thermodynamics (QGT), the RMS matter radii of ${ }^{4} \mathrm{He},{ }^{6} \mathrm{He}$ and ${ }^{8} \mathrm{He}$ nuclei, and the RMS charge radii of ${ }^{6} \mathrm{He}$ and ${ }^{8} \mathrm{He}$ nuclei with only one single input quantity (see Table 1). In this treatment the scale length underlying the system (in this case the proton size) is in principle an entirely free parameter that, once specified, defines the nuclear systems.

The other assumptions in this simple treatment are related to the weak binding of the systems, especially compared to the alpha-particle, which justifies the few-body description. The nuclear sizes are compared with experimental values from Tanihata et al. (1992) [ref.12] and Mueller et al. (2007) [ref.11]: Tanihata et al. measured RMS matter radii, with the neutron halo specifically distinguished by using the 2-neutron and 4-neutron removal cross-sections in collisions of an $800 \mathrm{MeV} /$ nucleon beam with a $\mathrm{C}$ target. These are the values adopted here, for the comparison with our calculations. Tanihata et al. also inferred the RMS neutron halo radii ( $10 \%$ larger than the RMS matter radii) from the fitted parameters of a Glauber optical model, but these radii are not used here. Mueller et al. measured RMS charge radii with small relative uncertainties via high precision laser spectroscopy of the isotope shifts in a magneto-optical cold trap. Their data were interpreted through the atomic theory of the Lamb shift ${ }^{34}$ for the low-lying S-states of $\mathrm{He}$ in order to deduce the RMS radius of the nuclear charge distribution.

We should comment that the value for the RMS matter radius of ${ }^{6} \mathrm{He}$ is not particularly well determined experimentally, despite the apparent clarity of the results in Table 1 (literature values are summarised as 2.45(10) fm after a detailed discussion, in the 2013 review of Tanihata $e t a l .{ }^{35}$ ). It is the dependence on the reaction model employed to interpret the experimental cross-section which is subject to the treatment of spatial correlations between the two neutrons and ${ }^{4} \mathrm{He}$ core that generates this uncertainty: this dependence was identified by al-Khalili et al. ${ }^{36}$ and explored more recently (with consistent conclusions) by Alkhazov \& Lobodenko [ref.29]. Both sets of authors deduce a value for the RMS radius of ${ }^{6} \mathrm{He}$ of at least $2.38 \mathrm{fm}$, with changes in the assumptions taking this as high as " $2.46 \mathrm{fm}$ or $2.70 \mathrm{fm}$ " (to 
underline this ambiguity). Thus, the results from QGT could be seen as amounting to an independent means of resolving these ambiguities.

The holomorphic treatment of ${ }^{6} \mathrm{He}$ can be compared to standard microscopic three-body calculations of the particle density distribution using a quantum mechanical model. These models often represent the probability density in terms of the Jacobian coordinates $r(n-n)$ and $R$ (nn-core), where $r$ is the distance between the halo neutrons and $R$ is the distance between the core $\left({ }^{4} \mathrm{He}\right.$ in this case) and the centre of mass of the halo neutrons. A typical example is Figure 4 of the 1996 review by Bang et al. [ref.20] which gives results for the ${ }^{6} \mathrm{He}$ ground state, and where the maximum densities are at $(R, r)=(2.6,1.0)$ and $(1.0,2.7) \mathrm{fm}$. Characteristically, there are two such peaks in probability density, generally referred to as the "dineutron" and the "cigar" solutions. Bang et al. report an RMS matter radius of "about $2.5 \mathrm{fm}$ (close to experiment)" from the calculations for ${ }^{6} \mathrm{He}$, which they call a "benchmark nucleus". The model of Hagino et al. [ref.26] shows a similar bimodal probability distribution in their description of the Borromean nucleus ${ }^{11} \mathrm{Li}$. In the three-body theoretical model of ${ }^{6} \mathrm{He}$ reported by Kikuchi et $a l .{ }^{37}$, the probability is plotted (in their Figure 2) as a function of two different variables that are more convenient for present purposes: that is, they show the probability (as a function of radius from the ${ }^{4} \mathrm{He}$ core) of finding the two halo neutrons in ${ }^{6} \mathrm{He}$ at that same radius and then also show in their two-dimensional plot the dependence of the probability on the angular separation between the two neutrons. Again, two peaks are found corresponding to the dineutron and cigar configurations, each peaking at a radius between 2 and $3 \mathrm{fm}$ and with angular separations of around $30^{\circ}$ and $135^{\circ}$ respectively. Note that their plot specifically requires the two neutrons to be at the same radius, whereas that of Bang et al. includes all configurations. The present treatment of ${ }^{6} \mathrm{He}$ insists on MaxEnt grounds that the correlation angle between the two halo neutrons is $2 \mathrm{p} / 3$ but we believe this should be interpreted as a representative average over all configurations

An analysis of the experimental data for ${ }^{6} \mathrm{He}$ by Bertulani \& Hussein $(2007)^{38}$ took into account measurements of the electric dipole strength $B(E 1)$ in dissociation experiments together with estimates of the source size in breakup experiments obtained via the Hanbury-Brown Twiss (HBT) interference. They deduced an average value of the opening angle between the halo nucleons of $83^{\circ}$ with an uncertainty of $\left(+20^{\circ},-10^{\circ}\right)$. However, Bertulani \& Hussein carefully acknowledge that the HBT analysis "was based on the use of a simple model of the emission of the two neutrons from a supposed random source" with the size (or existence) of "coherent effects" unknown. They also point out that "the $H B T$ probes the average $n-n$ configuration of the continuum states and not the ground state, as the nucleus is excited above the threshold before the emission occurs", which is of course the precondition for the $B(E 1)$ experiments. Bertulani \& Hussein also point out that their interpretations of the measurements are quite heavily model-dependent; indeed, the reason for introducing the HBT measurements was specifically to reduce this modeldependence. We emphasise that in our ab initio analysis of the ground state geometry the 
correlation angle of the halo neutrons is entailed by the charge radius, the measurement of which is quite unequivocal. Moreover, discussing the interesting and related case of ${ }^{11} \mathrm{Li}$, Hagino et al. [ref.26] show that the correlation angle is a function of the radius at which it is measured. The linear distance between the two neutrons (in their Figure 3a) is a minimum near the surface of the core and then scales with the absolute difference in the radial distance, from that point. This highlights questions about how the HBT results should be interpreted when translated into Bertulani \& Hussein's approach.

Table 2 is highly suggestive: we here comment on it only very briefly. Firstly, the QGT predictions that depend upon $\mathrm{D}^{1 / 2}$ are not so different from the usual close-packing or liquiddrop dependence of $r_{0} \mathrm{~A}^{1 / 3}$ over the range of masses up to $40 \mathrm{amu}$ (with $r_{0}=1.04 \mathrm{fm}$ ). It is therefore found that the whole series has a nuclear density very close to that of ${ }^{4} \mathrm{He}$ for a fixed scale length $\square_{\mathrm{H}}$. But the measured scale length $\square_{\mathrm{m}}$ (very similar to $\square_{\mathrm{H}}$ ) clearly varies systematically: it is not purely the holomorphic properties of the entropic geometry that determines nuclear size, the actual (kinematic) nuclear forces must also play a part. Recalling that an additional assumption (that the "He series" nuclear matter radii are quantised with the same length scale of $\square_{\mathrm{H}}$ as before) was required to produce reasonable values in Table 2 , it may be that this agreement has no great significance. However, the QGT account of the low nuclear density of ${ }^{8} \mathrm{Be}$ and ${ }^{10} \mathrm{He}$ explaining their instability does seem to be reasonable.

It is worth pointing out that Bohm's recognition of a "quantum-mechanical" potential $\mathrm{U}(x)$ exerting a "quantum-mechanical" force "analogous to, but not identical with the way in which ... a meson field exerts a force on a nucleon" (see his Eq.8) ${ }^{39}$, can be interpreted as an anticipation of our entropic force, discussed in a previous QGT treatment of galactic geometry (PJ2019, Eq.23). Bohm's proposal is considered by Rovelli ${ }^{40}$ to violate his Hypothesis 2 (of "completion"). However, neither author takes account of the entropic Principle of Least Exertion (PJ2019) in any way, despite each giving significant weight to the (physical) quantity Information in their different treatments. PJ2019 have shown that a physical system cannot be treated completely unless its info-entropy is also considered; moreover, the present Eqs.2, 3, 4 could be regarded as exemplifying Rovelli's Relational Quantum Mechanics, since the DoF numbers $\square=3,6,7$ are a direct consequence of observing the He core relative to its surrounds (including its halos). But a proper discussion of these issues is outside our scope.

\section{Conclusions}

In an ab-initio QGT analysis assuming a single scale length (the "holographic wavelength" $\square_{\mathrm{H}}$ given by twice the RMS proton radius), the nuclear matter radii and nuclear charge radii of the helium isotopes ${ }^{4,6,8} \mathrm{He}$ were calculated. These values are in remarkably good agreement with observation (Table 1).

In detail, the nuclear matter radii were calculated analytically by considering the holographic entropy of unitary and holomorphic entities as given by QGT, and noting that this entropy is proportional to the number of degrees of freedom (DoFs) of the entity. 
The appropriate number of DoFs for ${ }^{4} \mathrm{He}$, as a unitary entity in 3-space, is $\square \square=3$. The ${ }^{6} \mathrm{He}$ nucleus does not form a unitary object, but is formed by a sum of a unitary alpha core and a unitary neutron halo (of a holomorphic pair of neutrons); the two unitary entities being uncorrelated to each other apart from sharing a common centre. Consequently ${ }^{6} \mathrm{He}$ has $\square\left[=3+3=6\right.$ DoFs. On the other hand, the neutron halo of ${ }^{8} \mathrm{He}$ is also unitary, but formed of a holomorphic union of two holomorphic neutron pairs constrained to share a mean correlation angle, so that only a single additional $\mathrm{DoF}$ is gained. Consequently ${ }^{8} \mathrm{He}$ has $\square \square=3+(3+1)=7$ DoFs. From this information, combined with the holographic relationship between entropy and the system size, the RMS matter radii were determined and found to be in good agreement with experiment. The QGT approach will give a value for the RMS radius for any isotope as we continue to add pairs of neutrons and yet ${ }^{10} \mathrm{He}$ is known to be unbound, experimentally. To interpret this we suppose that QGT gives the size of the system if the internal forces allow it to be bound. The calculated sizes for ${ }^{10} \mathrm{He}$ and heavier isotopes of helium correspond to lower average nuclear densities that evidently are insufficient to keep the system bound.

The nuclear charge radii of these isotopes are determined geometrically using MaxEnt conditions imposed on the correlations between neutrons in the neutron halos. This works, despite the fact that such a geometric view cannot be strictly realistic for quantum objects.

The QGT approach can be extended to the nuclear matter radii of the "helium series" of nuclei, the self-conjugate $\mathrm{A}=4 n$ nuclei $\left\{{ }^{4} \mathrm{He},{ }^{8} \mathrm{Be},{ }^{12} \mathrm{C},{ }^{16} \mathrm{O},{ }^{20} \mathrm{Ne},{ }^{24} \mathrm{Mg},{ }^{28} \mathrm{Si},{ }^{32} \mathrm{~S},{ }^{36} \mathrm{Ar},{ }^{40} \mathrm{Ca}\right\}$. We find (for RMS matter radii calculated by QGT) that ${ }^{8} \mathrm{Be}$ should be to ${ }^{4} \mathrm{He}$ as ${ }^{6} \mathrm{He}$ is to ${ }^{4} \mathrm{He}$. That is, we expect ${ }^{8} \mathrm{Be}$ (considered as two alphas) to have $6 \mathrm{DoFs}$ like ${ }^{6} \mathrm{He}$, rather than the 4 DoFs that it would have if the two alphas formed a holomorphic pair. This implies that they should have the same entropic radius, and this prediction is consistent with the measured RMS radii. Similarly, ${ }^{12} \mathrm{C}$ is to ${ }^{8} \mathrm{Be}$ as ${ }^{8} \mathrm{He}$ is to ${ }^{6} \mathrm{He}$ : that is, both ${ }^{12} \mathrm{C}$ and ${ }^{8} \mathrm{He}$ have $7 \mathrm{DoFs}$ and the same entropic radius (also consistent with the measured radii). It then turns out that every step up the helium series simply adds one more DoF (see Table 2).

The nuclear sizes of both the helium series and the helium isotopes listed in Tables $\mathbf{1}, \mathbf{2}$ conform to a QGT treatment using a single uniform holographic wavelength: this is the only "free parameter", and is identified with the measured RMS proton size. It turns out that the sizes of the odd nuclei $\left\{{ }^{3} \mathrm{H},{ }^{7} \mathrm{Li},{ }^{11} \mathrm{~B},{ }^{15} \mathrm{~N},{ }^{19} \mathrm{~F},{ }^{23} \mathrm{Na},{ }^{27} \mathrm{Al},{ }^{31} \mathrm{P},{ }^{35} \mathrm{Cl},{ }^{39} \mathrm{~K}\right\}$ behave similarly with the same holographic wavelength. The case of ${ }^{6} \mathrm{He} \rightarrow{ }^{6} \mathrm{Li}$ appears to suggest that the beta decay process is associated with the acquisition of an extra DoF by the nucleus.

Tables $1 \& 2$ confirm that the nuclear sizes calculated from QGT both of He isotopes and of the "helium series" are realistic. This, together with previous quantitative work by Parker \& Jeynes [refs.1, 16, 17], indicates that this geometric entropy approach is valid for length scales over 35 orders of magnitude from sub-atomic to galactic.

As a unitary entity the a-particle is the simplest possible and can therefore be considered a "benchmark nucleus". The other isotopes of helium are also very simple nuclear structures in a 
QGT treatment: we expect these methods employing quantitative geometrical thermodynamics to have very wide application.

\section{Appendix 1: Terminology}

The term "holographic"- deriving originally from the Greek: o $\lambda \mathrm{o} \varsigma$ (whole) and $\gamma \rho \alpha \phi o \varsigma$ (written) - is used here in the same sense as the word hologram when it was introduced into physics by Gabor in 194941: "The name 'hologram' is not unjustified, as the photograph contains the total information required for reconstructing the object, which can be two-dimensional or threedimensional." Here we represent all of the information in a system in Minkowski four-space holographically as an object in three-space.

The term "holomorphic" was coined by mathematicians in the $19^{\text {th }}$ century in the context of the theory of analytic functions. Holomorphic functions are complex functions everywhere differentiable with finite differentials, while meromorphic functions have isolated points of non-analyticity (that is, poles) in the complex plane.

Roger Penrose (2004) ${ }^{42}$ has given a very general and quite extensive discussion of the relevance of holomorphic functions in physics; but Parker \& Walker $(2004)^{43}$ proved that information cannot be transmitted using holomorphic functions, a result central to the understanding of filters and the control of noise in networks and also critical to the development by Parker \& Jeynes (2019, "PJ2019") of a more systematic understanding of the relation of entropy and information. PJ2019 use the Holographic Principle to derive their entropy equations (cited here as Eqs.2), but further work [ref.17] has shown that this Principle can be derived from Liouville's Theorem (see also Appendix 4 here).

The Hilbert transform provides the theoretical underpinning (based on causality considerations) for the so-called "Kramers-Kronig relations" that feature widely in the physical sciences. These have a very large literature with recent applications (for example) in thermal insulation materials ${ }^{44}$, geophysics ${ }^{45}$, optics ${ }^{46}$, and astrophysics ${ }^{47}$. The Hilbert transform, the Kramers-Kronig relations, and holomorphic/meromorphic functions all depend on the analytical continuation properties of functions in the complex plane. Parker \& Jeynes (PJ2019) have proved that certain holomorphic geometries can be represented by entropic Lagrangian/Hamiltonian formulations that correctly obey the Cauchy-Riemann relations, and have consequently derived the Principle of Least Exertion (described by a variational calculus using the Euler-Lagrange equations) isomorphic to the Principle of Least Action, where "exertion" is the path integral of the entropic Lagrangian of dimensionality based on the Boltzmann constant $k_{\mathrm{B}}$ (isomorphic to "action", which is the path integral of the kinematic Lagrangian of dimensionality based on the Planck constant $\hbar$ ).

\section{Appendix 2: Degrees of Freedom}

Eqs.1,3,4 assert that the entropy of an entity is proportional to its number of degrees of freedom (DoFs) D, quantised by Boltzmann's constant $k_{\mathrm{B}}$ :

$$
S=\mathrm{D} \cdot k_{\mathrm{B}}
$$


Although it does not usually appear in this form, this can be understood as a standard result from the classical kinetic theory of gases, where the kinetic energy of a particle per $D o F$ is given by $E=1 / 2 m \dot{q}^{2}$, and $m$ is the particle inertial mass. Using the velocity of the gas molecule $\dot{q} \equiv \mathrm{d} q / \mathrm{d} t$ (where $q$ is the position variable of the conjugate $p, q$ pair in the kinematic Hamiltonian) as the key parameter of interest, the kinetic energy per unit velocity is $\mathrm{d} E / \mathrm{d} \dot{q}=m \dot{q}$ (i.e. kinematic momentum), whereas the entropy per unit velocity is given by $\mathrm{d} S / \mathrm{d} \dot{q}=k_{\mathrm{B}} / \dot{q}$, which is equivalent to the entropic momentum (see PJ2019 Eqs.9b\&9c).

That is to say, the kinetic entropy $T_{S}=m_{s} \ln q^{\prime}$ (see PJ2019 Eq.10b: $q^{\prime} \equiv \mathrm{d} q / \mathrm{d} x_{3}$ ) is differentially related to the entropic momentum via $\mathrm{d} T_{S} / \mathrm{d} q^{\prime}=m_{S} / q^{\prime}$, where the entropic mass $m_{S} \square \mathrm{i} k k_{\mathrm{B}}$ and $k$ is a real scale factor inversely proportional to the "holographic wavelength". The kinetic entropy and the entropic momentum are quantities completely isomorphic to the conventional kinematic quantities (see PJ2019 Table 1). Since entropy $S$ is the pathlength integral of the entropic Hamiltonian (PJ2019 Eq.14), we also exploit the Fourier differential operator $\mathrm{d} / \mathrm{d} x_{3} \square \mathrm{i} k$ (PJ2019 Eq.15) such that $S \square T_{S} / \mathrm{i} k$, resulting in $\mathrm{d} S / \mathrm{d} \dot{q}=k_{\mathrm{B}} / \dot{q}$, since $\dot{q}=c q^{\prime}$ where $c=\mathrm{d} x_{3} / \mathrm{d} t$ is the speed of light.

The thermodynamic temperature $\mathcal{T}$ is given by

$$
\mathcal{T}=\mathrm{d} E / \mathrm{d} S=(\mathrm{d} E / \mathrm{d} \dot{q}) /(\mathrm{d} S / \mathrm{d} \dot{q})=m \dot{q}^{2} / k_{\mathrm{B}}
$$

Rearranging gives an energy of $1 / 2 m \dot{q}^{2}=1 / 2 k_{\mathrm{B}} \cdot \mathcal{T}$ per DoF, or in 3 -space

$$
\text { Kinetic energy }=3 / 2 k_{\mathrm{B}} \mathcal{T}
$$

which is the standard result from the kinetic theory of gases; that is, each particle has $1 / 2 k_{\mathrm{B}} \mathcal{T}$ kinetic energy per degree of freedom.

Considering Eq.A.2.2a therefore indicates that we have $1 / 2 k_{\mathrm{B}}$ kinetic entropy per DoF. However, the entropic Hamiltonian $H_{S}$ requires the sum of both kinetic and potential entropies (which we note are both intrinsically geometric quantities) so that a spatial DoF has contributions from both types of entropy. Note that, from Nöther's theorem and the associated entropic Euler-Lagrange equations, the entropic Hamiltonian $H_{S}=T_{S}+V_{S}$ (PJ2019 Table 1) is a conserved quantity in QGT.

Now, for the case where a particle is located within a potential field, the potential energy $E_{V}$ is defined as $E_{V}=m \ddot{q} q$ where $m$ is the inertial mass, $q$ is the displacement in the potential field, and $\ddot{q}$ is the acceleration (the second derivative of $q$ with time; the dot indicates $\mathrm{d} / \mathrm{d} t$ as usual), with $\mathrm{d} E_{V} / \mathrm{d} q=m \ddot{q}$. Similarly, analysing the potential entropy and using primed variables to indicate $\mathrm{d} / \mathrm{d} x_{3}$ instead of the dot to indicate $\mathrm{d} / \mathrm{d} t$, the entropic potential is $V_{S}=m_{S}\left(q^{\prime \prime} / q^{2}\right) q$ (see PJ2019 Table 1) such that $\mathrm{d} V_{S} / \mathrm{d} q=m_{S}\left(q^{\prime \prime} / q^{2}\right)$. Here $m_{S}\left(\square \mathrm{i} k k_{\mathrm{B}}\right)$ is again the (complex) entropic mass. And since the associated potential entropy $S_{V}$ is the path integral of the entropic potential (with $\left.S_{V} \square V_{S} / \mathrm{i} k\right)$ we may write $\mathrm{d} S_{V} / \mathrm{d} q=k_{\mathrm{B}}\left(q^{\prime \prime} / q^{2}\right)$. Note that the kinematic and entropic formulae are isomorphic so that $\dot{q}$ can be replaced by $c q^{\prime}$ and $\ddot{q}$ can be replaced by $c^{2} q^{\prime \prime}$, and Eq.A2.2a can be replaced by:

$$
\mathcal{T}=\mathrm{d} E_{V} / \mathrm{d} S_{V}=\left(\mathrm{d} E_{V} / \mathrm{d} q\right) /\left(\mathrm{d} S_{V} / \mathrm{d} q\right)=m \dot{q}^{2} / k_{\mathrm{B}}
$$


Other things being equal, it is clear that in the context of a potential field the potential energy per DoF is seen to be equivalent to the kinetic energy per $\operatorname{DoF} 1 / 2 m \dot{q}^{2}$, such that we find that there is also $1 / 2 k_{\mathrm{B}} \mathcal{T}$ potential energy per DoF. Likewise, the associated potential entropy is therefore also equally $1 / 2 k_{\mathrm{B}}$ per DoF. This means that the overall entropy (kinetic + potential) associated with each spatial DoF is $k_{\mathrm{B}}$. Therefore, for 3-space:

$$
S=3 k_{\mathrm{B}}
$$

where Eq.A2.3 includes a factor 3 for the same reason that Eq.A2.2b does: both are representing the 3 DoFs available in 3-space.

This can also be seen by considering Eq.3.1 of Bousso [ref.3], who explicitly gives the number of degrees of freedom $\mathrm{D}$ of a quantum mechanical system as the logarithm of the size $\mathcal{N}$ of its Hilbert space $\mathcal{H}$ :

$$
\mathrm{D}=\ln \mathcal{N}=\ln \operatorname{dim}(\mathcal{H})
$$

in which $\ln \mathcal{N}$ looks similar to the $\ln W$ in Boltzmann's formula

$$
S=k_{\mathrm{B}} \ln W
$$

and since the state space size is given (in natural units) by $W=\mathcal{N}$, Eq.A2.1 again follows.

Our treatment of the alpha particle is comparable to that of a single atom in a monatomic ideal gas (yielding Eq.A2.3) since both types of particle are idealised as (MaxEnt) unitary objects; that is, with zero internal degrees of freedom.

The reason that a monatomic gas particle (atom) has three DoFs is ultimately because it exists in 3-D space. Interestingly, Pitre et al. (2017) ${ }^{48}$ have measured the Boltzmann constant $k_{\mathrm{B}}$ at the best available accuracy from the speed of sound in helium gas, and this is employed in the 2019 redefinition of the Système International d'unités ${ }^{49}$ to fix $k_{\mathrm{B}}$. Their Eq.1a is:

$$
v^{2}=5 / 3 \cdot R \mathcal{T} / M
$$

where $v$ is the speed of sound in an ideal gas, $R$ is the universal gas constant, $\mathcal{T}$ the absolute temperature and $M$ the molar mass. The "zero-density ratio of the constant-pressure specific heat to the constant-volume specific heat [is] exactly 5/3 for the monatomic gases" $" 50$ since it is given exactly by " $1+2 / f$ " where their $f$ (equivalent to our $\square$ ) is the number of DoFs of the particle: for a monatomic gas (like helium) $f=3$.

The phrase "ontological degrees of freedom" was used previously by 't Hooft $(2002)^{51}$ in the context of the information loss (at the Planck scale) suggested by his proposal (of dimensional reduction in quantum gravity). An "ontological state" for 't Hooft is a property of a measurable variable (a "be-able" in his deterministic model). We put a rather different (but related) construction on these ideas; in particular, our proposal, being entropic, is almost entirely scale-less, with DoFs (as well as things) explicitly manifesting themselves at all scales (including the nucleic scales of this paper). 
In this paper, the term "ontological degree of freedom" refers to the "freedom" of a contingent entity to either exist or not. In this sense, unity represents the minimum number of degrees of freedom of an existing thing. Were its number of DoFs zero, we could infer that the thing did not exist at all. With the thing (the unitary entity) possessing only a single degree of freedom (DoF, $\square=1$ ), it is clear that the thing has no choice about which state it is in. Each manifestation of a new term in the He series is therefore associated with an additional ontological DoF, as each new term (that is, new thing) building upon the existing thing (the previous term in the He series) via the addition of an alpha comes into existence. For more detail on this see Parker \& Jeynes, 2020 [ref.17].

In Eq.2b the scale of the nucleus is given implicitly by the holographic wavelength $\square_{\mathrm{H}}$, as is explicit in Eqs.6. Whether or not $\nabla_{\mathrm{H}}$ (given here by Eqs.5) can be determined absolutely ab initio is a separate question (for example, the entropy of black holes is known absolutely, as is acknowledged in the discussion of Eq.2b). See Appendix 3 for further discussion of this issue.

\section{Appendix 3: Info-Entropy}

The question of how Information and Entropy are related is covered comprehensively by Parker \& Jeynes [ref.1] (PJ2019) who show that they are Hodge duals of each other in exactly the same way that the electric and magnetic fields are mutually Hodge duals for field-free electromagnetic waves in the Riemann-Silberstein complex-vector (holomorphic) representation. Information is not simply negative entropy, as Brillouin stated. We say that Maximum Entropy (MaxEnt) implies minimum extra information, which is consistent with Brillouin's position.

For any given stable system we expect the entropy to be maximised since being MaxEnt implies stability. Stable systems do not have to be in thermal equilibrium: for example, the shape of a spiral galaxy is stable but galaxies are not in equilibrium since the central black hole must necessarily grow (this system is discussed at length in PJ2019). Stable non-equilibrium systems have non-zero potential entropy $V_{\mathrm{S}}$ whereas unitary systems have minimised their numbers of degrees of freedom and have therefore minimised $V_{\mathrm{S}}$.

Parker \& Jeynes [ref.17] have shown separately in considering the entropic Hamiltonian $H_{\mathrm{S}}$ (a conserved quantity which is the sum of the kinetic entropy $T_{\mathrm{S}}$ and the potential entropy $V_{\mathrm{S}}$, both derived by PJ2019 - see their Eqs.10,22) that as the number of degrees of freedom (DoFs) decreases so also does the potential entropy $V_{\mathrm{S}}$ (with a concomitant rise in the kinetic entropy $T_{\mathrm{S}}$ since $H_{\mathrm{S}}$ is conserved). The MaxEnt principle specifically maximises the kinetic entropy (given the potential entropy).

It is precisely because of this interchange between the DoFs (proportional to the potential entropy $V_{\mathrm{S}}$ ) of the system and the kinetic entropy $T_{\mathrm{S}}$ that the operation of entropic forces can be powerful generators of order [ref.5]. In this case we see the integrity of unitary entities guaranteed by their MaxEnt (that is, maximal kinetic entropy $T_{\mathrm{S}}$ ) status. 


\section{Appendix 4: Entropic Quantum Operators \& Holographic Entropy}

We give here an abbreviated introduction to QGT to indicate that the regular quantum operator representation follows directly for QGT from a proper treatment of the entropic Liouvillian. This is a more general (fundamental) approach underlying the treatment of PJ2019 which established the present Eqs.2. For more details see Ref.[17].

Following the standard treatment of Wannier, ${ }^{52}$ using the appropriate entropic density of states $\square_{S}\left(q_{1}, q_{2}, \ldots q_{N}, p_{1}, p_{2}, \ldots p_{N}, x_{3}\right)$, and using $x_{3}$ as the appropriate coordinate along the symmetry axis of the holomorphic structure (noting that $x_{3}$ therefore also has strong analogies with the more conventional time coordinate $t$; indeed they can be considered as mutually conjugate in the Pauli algebra (Ref.[1], App.A)), the entropic Liouville equation is given by:

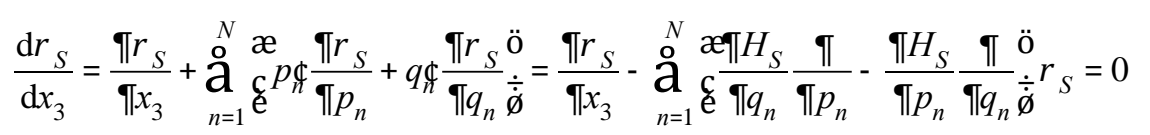

where $(p, q)_{n}$ are the entropic conjugate variables (entropic momentum and hyperbolic space, respectively) for the $n^{\text {th }}$ pair of degrees of freedom of the holomorphic system with overall $\mathrm{D}=2 N+1$ DoFs. The appropriate canonical equations of state are then given by the identities proved by PJ 2019 (Appendix B Eq.B17)

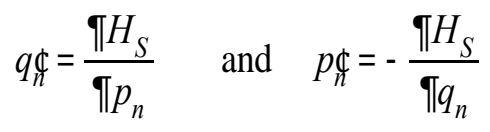

noting that the prime symbol indicates differentiation with respect to the $x_{3}$ co-ordinate:

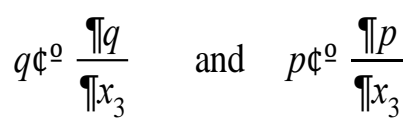

and where the entropic Hamiltonian $H_{S}$ depends on the maximum entropy (holomorphic) system as appropriate. So the fundamental structure is the logarithmic double-spiral, whose entropic Hamiltonian is given by PJ2019 (Appendix B Eq.B40b). The double helix is a special case of the logarithmic double-spiral: its entropic Hamiltonian is given by PJ2019 (Appendix B Eq.B19b). The sphere is a combination of double-helices, with an entropic Hamiltonian given by Parker \& Jeynes 2020 [ref.16] Eq.8a.

An elemental $2 N$-volume within the density of states $\square_{s}$ along the trajectory is given by $d \square s=\square_{n}\left(d p_{n} \square d q_{n}\right), \quad n \square\{1 \ldots N\}$; however in the special case of a simple MaxEnt (holomorphic) system which is specified by a single pair of entropic conjugate variables $(N=1)$, it is given by:

$$
d \square s=d p \square d q
$$

Wannier ([ref.52], Eq.3.07) shows that an alternative expression of Liouville's Theorem is that the logarithmic derivative of $d \square s$ at any point along the $x_{3}$ co-ordinate trajectory is zero, with $d \square s$ therefore being constant along the entropic trajectory:

$$
\frac{1}{d \mathrm{~W}_{S}} \frac{\mathrm{d}}{\mathrm{d} x_{3}}\left(d \mathrm{~W}_{S}\right)=\frac{\mathrm{d}}{\mathrm{d} x_{3}}\left(\ln d \mathrm{~W}_{S}\right)=0
$$

Then a unitary system where the degrees of freedom are minimised, that is a system with no subsystems at the entropic granularity of interest, will have an entropy simply given by: 


$$
S=k_{B} \ln M=\frac{\mathrm{W}_{S}}{\mathrm{D} p \mathrm{D} q} k_{B}
$$

where $k_{\mathrm{B}}$ is Boltzmann's constant as usual, and $M$ represents the total number of states across the entire phase space $\square$ s for a trajectory conforming to an entropy $S$. That is, the entropic phase space $\square_{s}$ is divided into and composed of cells of dimensionality $k_{\mathrm{B}}$ (in contrast to the conventional kinematic treatment where the phase space cells exhibit the Planck constant dimension $\hbar$ ). In fact, it can be shown that the Bekenstein-Hawking expression for the entropy of a Schwarzschild black hole (a unitary object) therefore also follows straightforwardly from Eq.A4.5. Indeed, it can be shown (see [ref.17]) that applying the geometry of the double-helix (as the unitary eigenvector of QGT) to Eq.A4.5 also enables the entropy $S$ to be expressed holographically as the ratio of the surface area $A$ enveloping the unitary system under consideration with the square of the associated granularity scale (holographic wavelength $\square_{\mathrm{H}}$ ) as per Eq.2b.

The entropic Liouvillian $\hat{\boldsymbol{L}}_{S}$ of such a conventional canonical Hamiltonian system is then :-

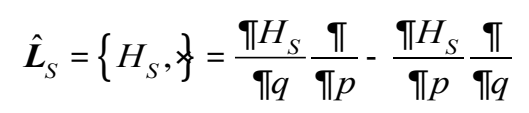

This may be transformed into a quantum system by replacing the Poisson brackets with the commutation operation and re-interpreting variables as quantum operators: then since $\mathrm{d} \nearrow_{s} / \mathrm{d} x_{3}=0$, we may write using Eq.A4.1 assuming a unitary $N=1$ structure:-

$$
\frac{\prod r_{S}}{\llbracket x_{3}}=\frac{-1}{2 k_{B}} \dot{\varepsilon} H_{S}, r_{S} \grave{\text { ù }}
$$

where of course the equivalent quantum Liouville equation for kinematics is $\square \square \square t=(1 / \mathrm{i} \hbar)[H, \square \square$ and the kinematical i $\hbar$ is isomorphic to the entropic $\square 2 k_{\mathrm{B}}$. The novel use of Boltzmann's constant in Eq.A4.7 represents a key difference from conventional quantum (kinematic) formalisms based upon Planck's constant. In other words, according to the Minkowski spacetime formalism, $k_{B}$ (associated with space-like quantities) and $\hbar$ (identified with time-like quantities) are orthogonal to each other, that is, they transform to each other by a $\square / 2$ rotation in the complex plane (hence the imaginary factor i).

The factor 2 that appears in Eq.A4.7 is also a reflection of an explicitly entropic version of the partition function being composed of probabilities that are analogous to the modulus-squared of the Schrödinger equation, e.g. as described by Córdoba et al. ${ }^{53}$ (their Eqs.29,30). For entropic systems (based on the conjugate variables $p_{S}$ and $q_{S}$, where we now here include the subscript ' $S$ ' to emphasise that these are entropic quantities) quantised using the Boltzmann constant, it is immediately apparent that the following entropic commutator result therefore also holds:

$$
\text { é } \hat{q}_{S}, \hat{p}_{S} \grave{\mathrm{q}}=-2 k_{B}
$$

This representation is the canonical commutation relation from which the entropic uncertainty principle immediately follows :-

$$
\mathrm{D} p \mathrm{D} q^{3} k_{B}
$$


It is also clear that in progressing from the classical Poisson brackets to the quantum entropic commutator, the entropic conjugate variable $p_{S}$ can be identified with the following entropic quantum operator:

$$
\hat{p}_{S} \stackrel{\mathrm{o}}{2} 2 k_{B} \frac{\mathbb{\mathbb { T }}}{\mathbb{T} q_{S}}
$$

Note that the entropic quantum operator (Eq.A4.10) is based in hyperbolic space $q_{S}$, whereas the conventional kinematic momentum operator $(p \equiv-\mathrm{i} \hbar \partial / \partial x)$ is based in Euclidean space $x$ (as is also the conventional Heisenberg uncertainty principle).

\section{Author contributions and Acknowledgments}

MCP developed the physics and wrote the paper; CJ critically discussed the physics, helped develop it, and wrote and edited the paper; WNC critically discussed the physics and edited the paper. Comments from Dr. N. K. Timofeyuk and extensive comments from Prof. I. J. Thompson are appreciated.

\section{Additional Information}

The authors declare no competing interests.

\section{References}

1 M.C.Parker, C.Jeynes, Maximum Entropy (Most Likely) Double Helical and Double Logarithmic Spiral Trajectories in Space-Time, Scientific Reports (2019) 9:10779 (10pp); http://dx.doi.org/10.1038/s41598-019-46765-w

2 E. T. Jaynes, Information theory and statistical mechanics. Physical Review, 106, 620-630 (1957)

3 Raphael Bousso, The Holographic Principle, Reports of Modern Physics 74 (2002) 825-874

4 Léon Brillouin, Science \& Information Theory (Academic Press, 1956).

5 Ilya Prigogine, The End of Certainty (The Free Press, New York 1996) ch.1 p.26

6 W.T.Grandy Jr, Time Evolution in Macroscopic Systems. II. The Entropy, Foundations of Physics, 34 (2004) 21-57

7 W.T.Grandy Jr, Entropy and the Time Evolution of Macroscopic Systems (OUP, 2008)

8 D.R. Tilley, C.M. Cheves, J.L. Godwin, G.M. Hale, H.M. Hofmann, J.H. Kelley, C.G. Sheu, H.R. Weller, Energy levels of light nuclei A=5, 6, 7, Nuclear Physics A 708 (2002) 3-163

9 D.R. Tilley, J.H. Kelley, J.L. Godwin, D.J. Millener, J.E. Purcell, C.G. Sheu, H.R. Weller, Energy levels of light nuclei A=8, 9, 10, Nuclear Physics A 745 (2004) 155-362

10 A. S. Jensen, K. Riisager, D. V. Fedorov, and E. Garrido, Structure and reactions of quantum halos, Reviews of Modern Physics 76 (2004) 215-261

11 P.Mueller, I.A.Sulai, A.C.C.Villari, J.A.Alcántara-Núñez, R.Alves-Condé, K.Bailey, G.W.F.Drake, M.Dubois, C.Eléon, G.Gaubert, R.J.Holt, R.V.F.Janssens, N.Lecesne, Z.-T.Lu, T.P.O’Connor, M.-G.SaintLaurent, J.-C.Thomas, L.-B.Wang, Nuclear charge radius of ${ }^{8} \mathrm{He}$, Physical Review Letters 99 (2007) 252501

12 I. Tanihata, D. Hirata, T. Kobayashi, S. Shimoura, K. Sugimoto and H. Toki, Revelation of thick neutron skins in nuclei, Physics Letters B 289 (1992) 261-266

13 Sabyasachi Kar, Y.K. Ho, Borromean binding in $\mathrm{H}$ with screened Coulomb potentials, Chemical Physics Letters 506 (2011) 282-285

14 Lucas A. Souza, Filipe F. Bellotti, Tobias Frederico, Wave function for two-neutron halo states, Journal of Physics: Conference Series 630 (2015) 012043 (6pp)

15 B. V. Danilin, N. B. Shul'gina, S.N.Ershov, and J. S. Vaagen, RNBT (Russian-Nordic-British Theory) Collaboration, Cluster Models of Light Nuclei and the Method of Hyperspherical Harmonics: Successes and Challenges, Physics of Atomic Nuclei, 72 (2009) 1272-1284 
16 M.C.Parker, C.Jeynes, Fullerene Stability by Geometrical Thermodynamics, ChemistrySelect 5 (2019) 5-14; http://dx.doi.org/10.1002/slct.201903633

17 M.C.Parker, C.Jeynes, Entropic Uncertainty Principle, Partition Function and Holographic Principle derived from Liouville's Theorem, PhysicsOpen (2020, under review)

18 Jennifer Coopersmith, The Lazy Universe: an Introduction to the Principle of Least Action (OUP, 2017)

19 M.V. Zhukov, B.V. Danilin, D.V. Fedorov, J.M. Bang, I.J. Thompson, J.S. Vaagen, Bound state properties of Borromean halo nuclei: ${ }^{6} \mathrm{He}$ and ${ }^{11} \mathrm{Li}$, Physics Reports 231 (1993) 151-199

20 J.M.Bang, Russian-Nordic-British Theory (RNBT) collaboration: B.V.Danilin, V.D.Efros, J.S.Vaagen, M.V.Zhukov, I.J.Thompson, Few-body aspects of Borromean halo nuclei, Physics Reports 264 (1996) 27-37

21 C. Howson, P. Urbach, Scientific Reasoning: The Bayesian Approach (Open Court, 1989) $3^{\text {rd }}$ ed. (2006) Ch. 9, p.277

22 Peter J. Mohr, David B. Newell, Barry N. Taylor, CODATA recommended values of the fundamental physical constants: 2014, Journal of Physical and Chemical Reference Data 45 (2016) 043102 (74 pp)

23 Randolf Pohl and the CREMA Collaboration (33 authors), Laser spectroscopy of muonic deuterium, Science 363 (2016) 669-673

24 Carl E. Carlson, The proton radius puzzle, Progress in Particle and Nuclear Physics 82 (2015) 59-77

25 W. Xiong, A. Gasparian et al. (58 authors from 17 institutes), A small proton charge radius from an electron-proton scattering experiment, Nature 575 (2019) 147-151

26 K. Hagino, H. Sagawa, J. Carbonell, P. Schuck, Coexistence of BCS- and BEC-Like Pair Structures in Halo Nuclei, Physical Review Letters 99 (2007) 022506 (4pp)

27 Y.Kubota et al., Surface localization of the dineutron in ${ }^{11} \mathrm{Li}$, Physical Review Letters (accepted $8^{\text {th }}$ October 2020)

28 A. Ozawa, T. Suzuki, I. Tanihata, Nuclear size and related topics, Nuclear Physics A 693 (2001) $32-62$

29 G. D. Alkhazov, A. A. Lobodenko, Reaction Cross Sections for Collisions Involving Exotic Light Nuclei within the Glauber Approach, Physics of Atomic Nuclei 70 (2007) 93-108.

30 Yu.L. Parfenova, Study of Exotic Nuclei in the Flerov Laboratory of Nuclear Reactions at Joint Institute for Nuclear Research, Acta Polonica 49 (2018) 495-502

31 I.Angeli, Table of nuclear root-mean-square charge radii, IAEA-INDC, Vienna, September 1999

32 Akihiro Tohsaki, Hisashi Horiuchi, Peter Schuck, Gerd Röpke, Colloquium: Status of $\alpha$-particle condensate structure of the Hoyle state, Reviews of Modern Physics 89 (2017) 011002 (16 pp.)

33 J. Bishop, Tz. Kokalova, M. Freer, L. Acosta, M. Assié, S. Bailey, G. Cardella, N. Curtis, E. De Filippo, D. Dell'Aquila, S. De Luca, L. Francalanza, B. Gnoffo, G. Lanzalone, I. Lombardo, N. S. Martorana, S. Norella, A. Pagano, E. V. Pagano, M. Papa, S. Pirrone, G. Politi, F. Rizzo, P. Russotto, L. Quattrocchi, R. Smith, I. Stefan, A. Trifirò, M. Trimarchì, G. Verde, M. Vigilante, C. Wheldon, Experimental investigation of $\alpha$ condensation in light nuclei, Physical Review C 100 (2019) 034320 (20 pp)

34 G. W. F. Drake, Helium. Relativity and QED, Nuclear Physics A 737 (2004) 25-33

35 Isao Tanihata, Herve Savajols, Rituparna Kanungo, Recent experimental progress in nuclear halo structure studies, Progress in Particle and Nuclear Physics 68 (2013) 215-313

36 J. S. Al-Khalili, J. A. Tostevin, and I. J. Thompson, Radii of halo nuclei from cross section measurements, Physical Review C 54 (1996) 1843-1852

37 Yuma Kikuchi, Kazuyuki Ogata,Yuki Kubota, Masaki Sasano, and Tomohiro Uesaka, Determination of a dineutron correlation in Borromean nuclei via a quasi-free knockout (p, pn) reaction, Progress of Theoretical and Experimental Physics 10 (2016) 103D03 (14 pp)

38 C. A. Bertulani and M. S. Hussein, Geometry of Borromean halo nuclei, Physical Review C 76 (2007) 051602(R) (4pp)

39 David Bohm, A suggested interpretation of the Quantum Theory in terms of hidden variables I, Physical Review 85 (1952) 166-179

40 Carlo Rovelli, Relational Quantum Mechanics, International Journal of Theoretical Physics 35 (1996) 1637-1678

41 Dennis Gabor, Microscopy by reconstructed wave-fronts, Proceedings of the Royal Society A 197 (1949) 454-487

42 Roger Penrose, The Road to Reality (Jonathan Cape 2004).

43 M.C.Parker, S.D.Walker, Information transfer and Landauer's principle, Optics Communications 229 (2004) 23-27

44 Jianming Yang, Huijun Wua, Moran Wang, Yuying Liang, Prediction and optimization of radiative thermal properties of nano $\mathrm{TiO}_{2}$ assembled fibrous insulations, International Journal of Heat and

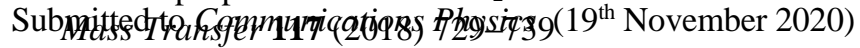


45 Yufeng Wang, Xiong Ma, Hui Zhou, Yangkang Chen, $\mathrm{L}_{1-2}$ minimization for exact and stable seismic attenuation compensation, Geophysical Journal International 213 (2018) 1629-1646

46 Pavel Peterka, Pavel Koška, Jiř́ Čtyroký, Reflectivity of Superimposed Bragg Gratings Induced by Longitudinal Mode Instabilities in Fiber Lasers IEEE J. Selected Topics in Quantum Electronics 24 (2018) 0902608 (8pp)

47 Daniel Kitzmann, Kevin Heng, Optical properties of potential condensates in exoplanetary atmospheres, Monthly Notices of the Royal Astronomical Society 475 (2018) 94-107

48 L Pitre, F Sparasci, L Risegari, C Guianvarc'h, C Martin, M E Himbert, M D Plimmer, A Allard, B Marty, P A Giuliano Albo, B Gao, M R Moldover, J B Mehl, New measurement of the Boltzmann constant $k$ by acoustic thermometry of helium-4 gas, Metrologia 54 (2017) 856-873

49 Joachim Fischer, The Boltzmann Constant for the Definition and Realization of the Kelvin, Annalen der Physik (Berlin), 531 (2019) 1800304 (12pp)

50 M R Moldover, R M Gavioso, J B Mehl, L Pitre, M de Podesta, J T Zhang. Acoustic gas thermometry, Metrologia 51 (2014) R1-R19

51 Gerard 't Hooft, Determinism beneath Quantum Mechanics, arXiv: quant-ph/0212095, (2002). Talk presented at: Workshop on Philosophy of Quantum Theory, Temple University, Philadelphia, Sep.2002. Quo Vadis Quantum Mechanics? (Eds. A.Elitzur, S.Dolev, N.Kolenda) Frontiers Collection (2005) 99-111

52 G.H. Wannier, "Statistical Physics", Dover, §3-1 (1966)

53 P Fernández de Córdoba, J M Isidro and Milton H Perea, Emergence from irreversibility, Journal of Physics: Conference Series 442, 012033 (2013, 8pp) 